\title{
Emergency Autotransfusion for Managing latrogenic Hemorrhagic Pericardial Effusion
}

\author{
Muhammad Alia, b, Stephan Behrend ${ }^{\mathrm{a}}$, Stefan Andreas Lange ${ }^{\mathrm{a}}$
}

\begin{abstract}
Iatrogenic hemorrhagic pericardial effusion (IHPE) is one of the major complications encountered in daily percutaneous intracardiac interventions. A 79-year-old man was scheduled for percutaneous left atrial appendage closure (PLAAC) at our department. He had nonvalvular atrial fibrillation, with a contraindication to oral anticoagulants because of a history of recurrent significant intestinal bleeding with angiodysplasia. During the deployment of the PLAAC device (Watchman device), patient became hemodynamically unstable with a typical decrease in the systolic arterial pressure of more than 10 $\mathrm{mm} \mathrm{Hg}$ during inspiration (pulsus paradoxus) because pericardial tamponade occurred due to perforation of the left atrial appendage. We report our successful experience with management of IHPE by immediate pericardiocentesis, insertion of percutaneous catheter drainage (PCD), and retransfusing drained pericardial blood through a central venous line. IHPE is not uncommon complication in daily percutaneous intracardiac interventions. Pulsus paradoxus is the most important clinical sign of cardiac tamponade. Our approach by immediately retransfusing drained pericardial blood through a central venous line allowed rapid physiologically appropriate recovery. Future consensus of opinion of experts focusing on autotransfusion in such cases is needed.
\end{abstract}

Keywords: Pericardial effusion; Left atrial appendage; Autotransfusion; Intracardiac intervention

\section{Introduction}

Iatrogenic hemorrhagic pericardial effusion (IHPE) is one of the major complications of the percutaneous left atrial appendage closure (PLAAC) procedure [1]. It occurs in about $3.8 \%$ of patients who undergo left atrial appendage closure (LAAC) with the Watchman device [1]. About $89 \%$ of patients with IHPE develop hemodynamic deterioration and require in-

Manuscript accepted for publication March 17, 2017

aDepartment of Cardiology, Asklepios Harzklinik, Goslar, Germany

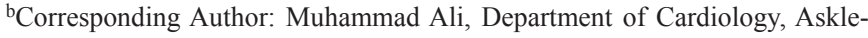
pios Harzklinik Goslar, Kosliner Str. 12, 38640 Goslar, Germany. Email:m.ali7@icloud.com

doi: https://doi.org/10.14740/jmc2789w tervention within $24 \mathrm{~h}$ of the procedure [1]. Iatrogenic acute cardiac tamponade is a life-threatening complication that can lead to death; it often involves hemodynamic instability, and requires cardiopulmonary resuscitation in $20 \%$ of patients and blood transfusion in more than $25 \%$ of patients [2]. When IHPE occurs, any concomitant intracardiac procedure should be cancelled, and anticoagulation should be reversed [3]. These two steps are sufficient if the pericardial effusion is small and not hemodynamically significant $[1,3]$. In cases of tamponade, pericardiocentesis and percutaneous catheter drainage (PCD) should be urgently performed, and in certain circumstances, surgical repair is required $[1,3]$.

Traditionally, the drained pericardial blood is collected in a drainage bag, and an allogenic blood transfusion is used to correct low hemoglobin levels in patients with significant bleeding who are hemodynamically unstable [3]. However, blood transfusions may carry some risk of complications.

We describe our experience with managing IHPE by immediately retransfusing drained pericardial blood through a central venous line.

\section{Case Report}

A 79-year-old man was scheduled to undergo LAAC in our department. He had non-valvular atrial fibrillation with a contraindication to oral anticoagulants because of a history of recurrent significant intestinal bleeding with angiodysplasia, as well as an increased risk of bleeding (HAS-BLED score, $5)$. He also had a high risk for thromboembolism $\left(\mathrm{CHA}_{2} \mathrm{DS}_{2}-\right.$ VASc score, 4).

He was sedated with propofol $2 \%$, and spontaneous respiration was maintained. All standard anesthetic monitors were attached. Invasive blood pressure monitoring was performed using a 4-French sheath inserted into the left femoral artery. Unfractionated heparin (70 units/ $\mathrm{kg}$, intravenous bolus) was administered at the beginning of the procedure to achieve a target activated clotting time of 250 - $300 \mathrm{~s}$. During manipulation of the stiff wire and deployment of the LAAC device (Watchman device $27 \mathrm{~mm}$ ) in the LAA, he became hemodynamically unstable; the intra-arterial pressure decreased suddenly, from $125 / 80$ to $84 / 33 \mathrm{~mm} \mathrm{Hg}$, with a typical decrease in the systolic arterial pressure of more than $10 \mathrm{~mm} \mathrm{Hg}$ during inspiration (pulsus paradoxus) (Fig. 1). His heart rate increased from 62 to 115 beats/min. Additionally, bilateral jugular venous distension was noted. An echocardiogram demonstrated pericardial effusion with diastolic compression of the right ventricle and 


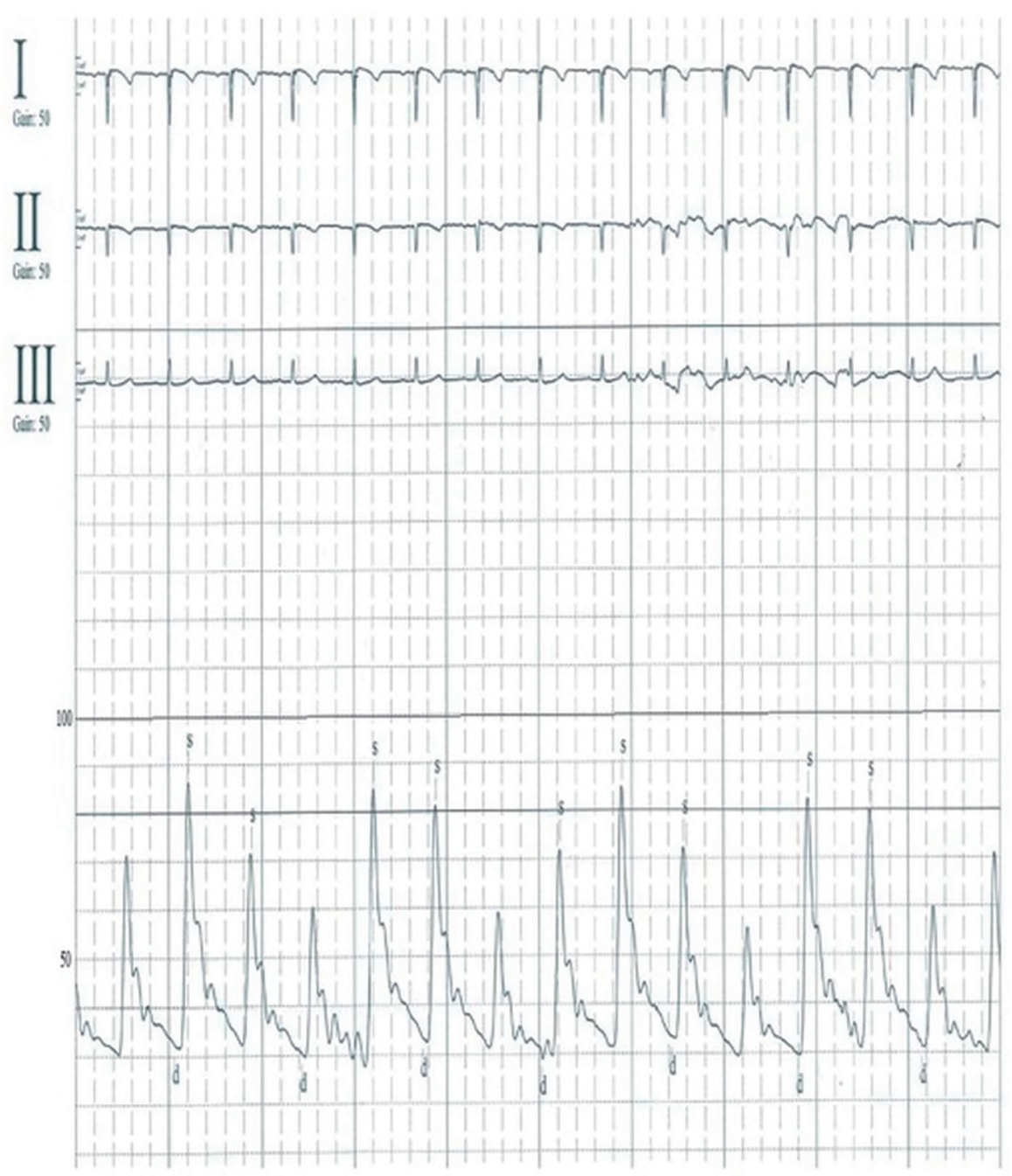

Figure 1. Patients' electrocardiogram findings and arterial blood pressure, which was monitored using an arterial catheter. Pulsus paradoxus is present, as the systolic intra-arterial pressure decreased by $>10 \mathrm{~mm} \mathrm{Hg}$ during aspiration.

right atrium (Fig. 2). Subxiphoid pericardiocentesis was immediately performed using ultrasound guidance. A 5-French pigtail catheter was inserted into the pericardium, after which we aspirated blood from the pericardium by using a $50-\mathrm{mL}$ syringe. Then we reinfused the blood through a 5-French central venous catheter into the right femoral vein. This action was repeated approximately 50 times. Parallel colloidal fluids were rapidly infused. An angiogram showed contrast agent in the pericardium with perforation of the LAA (Fig. 3). The procedure was terminated, and a suitable dose of protamine sulphate was administered to reverse the effects of heparin. Pericardial blood aspiration and reinfusion resulted in a noticeable improvement in the patient's hemodynamic status, and bleeding from the LAA stopped. The patient was transferred to the intensive care unit in a hemodynamically stable condition. Follow-up echocardiograms showed no evidence of pericardial effusion, and the pigtail catheter was removed after $24 \mathrm{~h}$. The patient was asymptomatic and had a good clinical condition; hence, he was discharged from the hospital on the fifth day postoperatively. He was informed in detail about the complication, and he decided to receive medical treatment with clopidogrel.

\section{Discussion}

In the setting of IHPE, clinical, echocardiographic, and radiologic evaluations are essential. Clearly, making an early diagnosis and having an experienced interventional cardiologist perform emergency pericardiocentesis are life-saving measures in cases of tamponade $[4,5]$.

The present report describes an emergency response to a nearly catastrophic complication of PLAAC, wherein pericardial tamponade was reversed by aspiration of the pericardial hemorrhage, followed by immediate reinfusion of that blood. Our patient received his own blood, which is safer, easier, and more cost-effective, and it contains more adequate clotting factors and platelets than banked blood. On the basis of our 


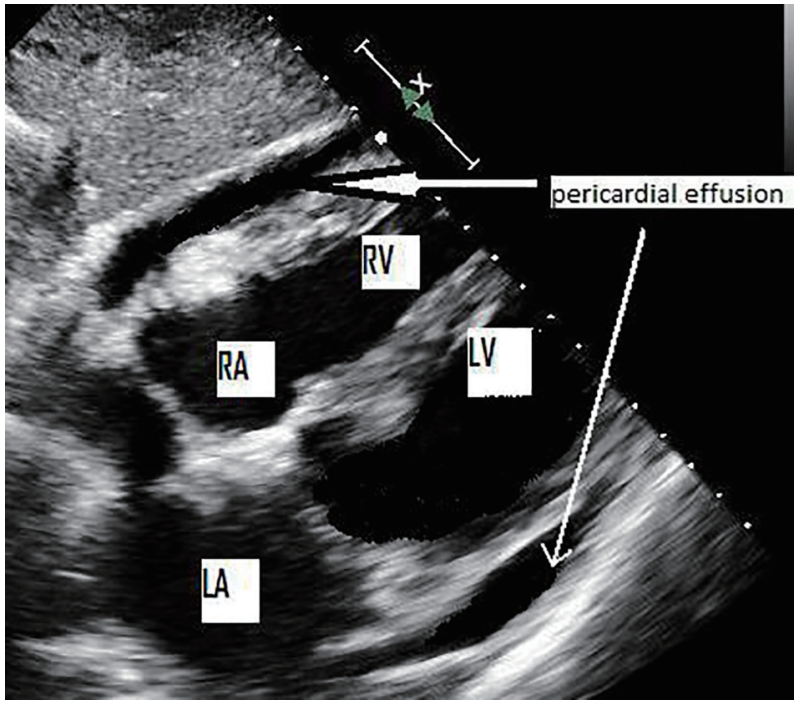

Figure 2. Echocardiographic view showing pericardial effusion with partially diastolic compression of the right atrium and right ventricle. RA: right atrium; RV: right ventricle; LA: left atrium; LV: left ventricle.

experience, we recommend gently aspirating blood from the pericardium and directly reinjecting that blood through a large central venous line. We do not advise retransfusing collected blood from the drainage bag to avoid complications such as thrombosis, air embolism, and hemolysis. We believe that our experience makes a significant contribution to the literature because this complication is commonly encountered in percutaneous intracardiac interventions, and our approach facilitated rapid yet physiologically appropriate recovery. Additionally, our report is the first in the medical literature about a new successful strategy for treating IHPE by using autotransfusion.

\section{Conclusion}

IHPE is not uncommon complication in daily percutaneous intracardiac interventions. Pulsus paradoxus is the most important clinical sign of cardiac tamponade. Our approach to reinfusion of the drained pericardial blood may allow rapid physiologically appropriate recovery. There are currently no guidelines on the significance of autotransfusion in the treatment of IHPE patients. Future consensus of opinion of experts focusing on autotransfusion in such cases is needed.

\section{Acknowledgments}

We thank Elsevier's Webshop for editing the manuscript.

\section{Funding}

None.

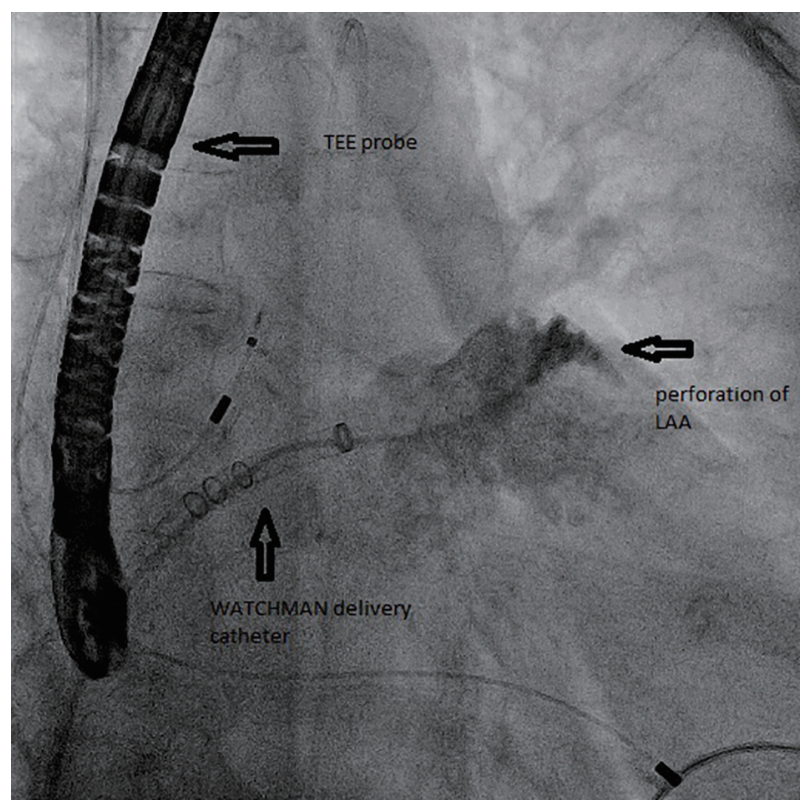

Figure 3. Fluoroscopy demonstrating contrast agent in the pericardium with perforation of the left atrial appendage during deployment of the Watchman device. TEE: transesophageal echocardiogram.

\section{Conflicts of Interest}

None.

\section{References}

1. Reddy VY, Holmes D, Doshi SK, Neuzil P, Kar S. Safety of percutaneous left atrial appendage closure: results from the Watchman Left Atrial Appendage System for Embolic Protection in Patients with AF (PROTECT AF) clinical trial and the Continued Access Registry. Circulation. 2011;123(4):417-424.

2. Bunch TJ, Asirvatham SJ, Friedman PA, Monahan KH, Munger TM, Rea RF, Sinak LJ, et al. Outcomes after cardiac perforation during radiofrequency ablation of the atrium. J Cardiovasc Electrophysiol. 2005;16(11):11721179.

3. Holmes DR, Jr., Nishimura R, Fountain R, Turi ZG. Iatrogenic pericardial effusion and tamponade in the percutaneous intracardiac intervention era. JACC Cardiovasc Interv. 2009;2(8):705-717.

4. Tsang TS, Enriquez-Sarano M, Freeman WK, Barnes ME, Sinak LJ, Gersh BJ, Bailey KR, et al. Consecutive 1127 therapeutic echocardiographically guided pericardiocenteses: clinical profile, practice patterns, and outcomes spanning 21 years. Mayo Clin Proc. 2002;77(5):429-436.

5. Cooper JP, Oliver RM, Currie P, Walker JM, Swanton RH. How do the clinical findings in patients with pericardial effusions influence the success of aspiration? $\mathrm{Br}$ Heart J. 1995;73(4):351-354. 